\title{
PAH EVOLUTION IN THE HARSH ENVIRONMENT OF THE ISM
}

\author{
H. Kaneda ${ }^{1}$, T. Onaka ${ }^{2}$, I. Sakon ${ }^{2}$, D. Ishihara ${ }^{1}$, A. Mouri ${ }^{1}$, \\ M. Yamagishi ${ }^{1}$ and A. Yasuda ${ }^{1}$
}

\begin{abstract}
We review observational results of PAH emission in harsh interstellar environments, which are mostly based on recent works by Spitzer and AKARI. The harsh environments include shock regions in our Galaxy, the ionized superwinds and haloes of external galaxies, and the hot plasmas of elliptical galaxies. Owing to the unprecedented high sensitivity for PAH emission with Spitzer and AKARI, it is found that an appreciable amount of PAHs are present in some cases with such hostile conditions. Some of them exhibit unusual PAH interband strength ratios, reflecting either evolution of PAHs or physical conditions of the ISM. The distribution of the PAH emission, as compared to that of dust emission, is shown to discuss their ways of evolution and survival.
\end{abstract}

\section{Introduction}

The evolution and survival of polycyclic aromatic hydrocarbons (PAHs) in the harsh environments of the interstellar medium (ISM) are interesting and important problems to be addressed for understanding the lifecycle of PAHs in space. PAHs are expected to be processed and destroyed quite easily in interstellar shock regions and hot plasmas (e.g. Tielens 2008). In general, as observed in our Galaxy and nearby galaxies, most PAHs are associated with neutral gas and their emission is weak in ionized regions with strong radiation field probably due to destruction (e.g. Boulanger et al. 1988; Désert et al. 1990; Bendo et al. 2008). However the true destruction efficiency of PAHs has not yet been well understood from astronomical observations because of scarcity of relevant data and their faintness in the mid-IR emission in such conditions, although there are several theoretical studies (Jones et al. 1996; Micellota et al. 2010a, 2010b). Now the situation has been

\footnotetext{
${ }^{1}$ G. School of Science, Nagoya University, Chikusa-ku, Nagoya 464-8602, Japan

${ }^{2}$ Graduate School of Science, The University of Tokyo, Bunkyo-ku, Tokyo 113-0033, Japan
} 
improved with the advent of Spitzer and AKARI; owing to their unprecedented high sensitivity for PAH emission, it is found that PAHs are indeed destroyed quite efficiently as compared to dust grains in most objects with such hostile conditions, but in some cases, an appreciable amount of PAHs are still present. Some of them exhibit unusual PAH interband strength ratios, reflecting either evolution of PAHs or physical conditions of the ISM.

From recent theoretical studies combined with laboratory data, Micellota et al. (2010a, 2010b) calculated the collisional destruction efficiency of PAHs in interstellar shocks and hot plasma. According to their results, for shock velocities lower than $100 \mathrm{kms}^{-1}$, PAHs in shocks are not completely destroyed but their structures are significantly affected. In such a slow shock, PAHs may even be formed by shattering of carbonaceous grains. On the other hand, for shock velocities higher than $200 \mathrm{~km} \mathrm{~s}^{-1}$, PAHs are completely destroyed in postshock hot plasma.

In hot plasmas with temperatures lower than $3 \times 10^{4} \mathrm{~K}$, ion nuclear collisions dominate in the destruction process, which destroy PAHs not so efficiently; for example, in a hot plasma with the density and temperature of $10^{4} \mathrm{~cm}^{-3}$ and $10^{4} \mathrm{~K}$ (e.g. Orion), small (50-200 C-atom) PAHs can survive against the collisional destruction for a time as long as $10^{7}$ years. For hotter plasma, however, electron collisions dominate in the destruction process, which destroy PAHs very efficiently. For example, in a hot plasma with the density and temperature of $10^{-2} \mathrm{~cm}^{-3}$ and $10^{7} \mathrm{~K}$ (e.g. M 82 superwinds), small PAHs can survive only for 100 years agaist collisional destruction (Micellota et al. 2010b). It should be noted that these lifetimes of PAHs are two to three orders of magnitude shorter than those for dust grains with the same size, because the sputtering yields of 3-dimensional dust grains are much smaller than unity, while the dissociation yields of 2-dimentional PAHs are close to unity. In HII regions with strong UV radiation field, the photo-dissociation of PAHs is more important, where the lifetimes of PAHs are $\sim 10^{5}$ year for typical HII regions while they are $\sim 10^{8}$ years for the diffuse ISM (Allain et al. 1996).

Below, we review observational results of PAH emissions in harsh interstellar environments, which are mostly based on recent works by Spitzer and AKARI. The harsh environments include shock around SNRs and intense UV field in a massive cluster in our Galaxy, the ionized superwinds and haloes of external galaxies, and the hot plasmas of elliptical galaxies.

\section{PAHs in harsh environments in our Galaxy}

According to Spitzer studies of PAH emission from Galactic SNRs by Reach et al. (2006), only 4 SNRs, out of 95, indicate the presence of PAH emission from IRAC colors. One example is $3 \mathrm{C} 396$, where the $8 \mu \mathrm{m}$ PAH map shows the presence of filamentary structures inside the radio shell. The Spitzer IRS also detected PAH emission features from the spectra of the SNRs W28, W44, and 3C 391 (Neufeld et al. 2007), but their association with the SNRs was not so clear. For the SNR N132D in the Large Magellanic Cloud (LMC), a PAH $17 \mu \mathrm{m}$ broad feature was detected from Spitzer/IRS spectra of the southern rim across the shock front 


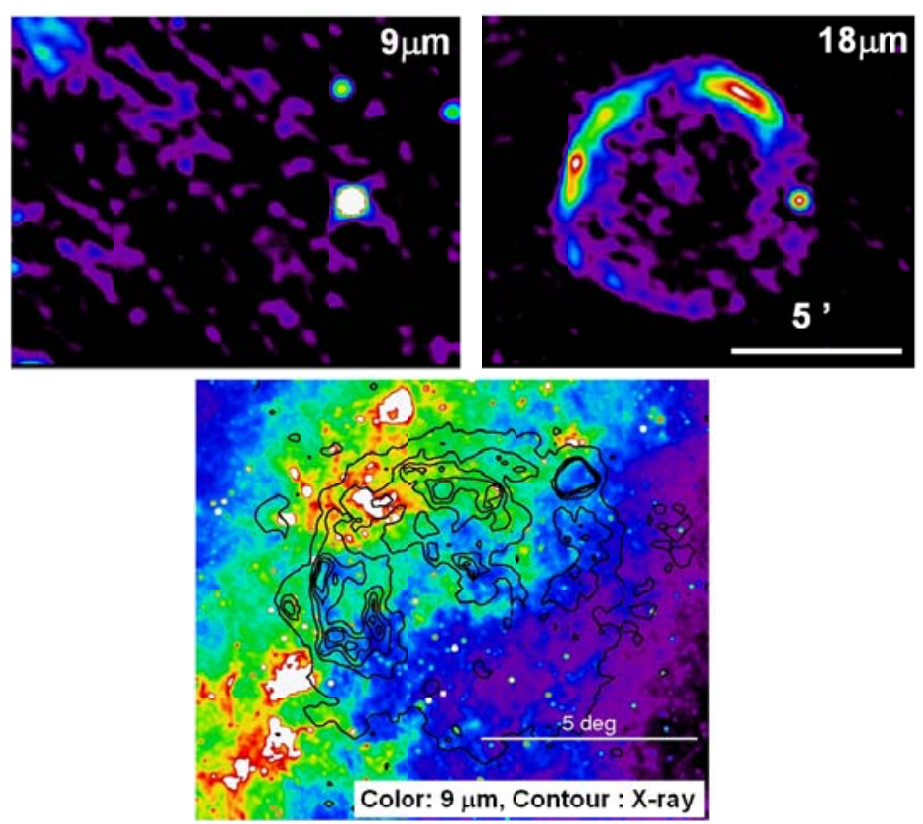

Fig. 1. AKARI $9 \mu \mathrm{m}$ and $18 \mu \mathrm{m}$ images of the Tycho SNR on the left and right of the upper panel, respectively (Ishihara et al. 2010), and the AKARI $9 \mu \mathrm{m}$ large-scale image around the Vela SNR in the lower panel, which are created from the AKARI mid-IR all-sky survey data. The ROSAT X-ray contours are superposed on the Vela SNR image.

(Tappe et al. 2006). The $17 \mu \mathrm{m}$ broad feature is interpreted as the emission of larger PAHs with a few thousand carbon atoms in $\mathrm{C}-\mathrm{C}-\mathrm{C}$ in- and out-of-plane bending modes (Van Kerckhoven et al. 2000; Peeters et al. 2004). The lifetime of large (4000 C-atom) PAHs is estimated to be $\sim 200$ years (Micellota et al. 2010b) while the age of the SNR is $\sim 2500$ years, and therefore such large PAHs may have been recently swept up by the blast wave and not yet completely destroyed by the shock (Tappe et al. 2006).

AKARI mid-infrared (IR) all-sky survey data are useful to discuss the presence of PAHs in SNRs. They consist in photometry in two wide bands centered at wavelengths of $9 \mu \mathrm{m}$ and $18 \mu \mathrm{m}$ with effective wavelength ranges of $6.7-11.6 \mu \mathrm{m}$ and 13.9-25.6 $\mu \mathrm{m}$, respectively (Onaka et al. 2007). The $9 \mu \mathrm{m}$ band includes the PAH features at 7.7, 8.6, and $11.3 \mu \mathrm{m}$, while the $18 \mu \mathrm{m}$ band traces warm dust continuum emission. The AKARI $9 \mu \mathrm{m}$ band is relatively broad as compared to the MSX and Spitzer bands for PAH emission, and it is therefore more likely to be dominated by PAH features and less affected by emission lines.

Structures associated with SNRs are often identified in the AKARI $18 \mu \mathrm{m}$ band, whereas they are not in the AKARI $9 \mu \mathrm{m}$. For example, the upper panel of Figure 1 shows the AKARI $9 \mu \mathrm{m}$ and $18 \mu \mathrm{m}$ images of the Tycho SNR at an age 
of $\sim 440$ years, where a spherical shell structure is clearly seen in the warm dust continuum emission while it is hardly seen in the PAH emission. The dust emission shows a beautiful spatial correlation with the X-ray and CO emission (Ishihara et al. 2010). With the current shock speeds ( $3000 \mathrm{~km} \mathrm{~s}^{-1}$; Reynoso et al. 1997) and the thickness of the dust emission region, we estimate dust residence time in postshock plasma to be $\sim 50$ years, where the X-ray plasma has temperature and electron density of $8 \times 10^{6} \mathrm{~K}$ and $10 \mathrm{~cm}^{-3}$, respectively (Warren et al. 2005). Then Figure 1 demonstrates that the dust can survive while the PAHs are almost totally destroyed in the 50 year timescale in a hot plasma with the above plasma parameters, which is consistent with the theoretical prediction. The lower panel of Figure 1 shows an AKARI $9 \mu \mathrm{m}$ image around the Vela SNR $\left(\sim 1.1 \times 10^{4}\right.$ years old), on which the X-ray contours are superposed. In this large-scale image, we can clearly see an anti-correlation between the PAH and the X-ray emission, again demonstrating effective destruction of PAHs in the postshock hot plasma.

Other than SNRs, AKARI also reveals the properties of PAHs in harsh environments of clouds with energetic phenomena with the help of large-scale CO data by the NANTEN telescope. One example is RCW49, Wd2, one of the most massive star clusters in our Galaxy. The massive cluster formation was likely triggered by cloud-cloud collision $\sim 4 \times 10^{6}$ years ago because the NANTEN CO observations revealed two large molecular clouds with a systematic difference in the speed $\left(\sim 10 \mathrm{~km} \mathrm{~s}^{-1}\right)$, the crossing point of which exactly corresponds to the position of Wd2 (Furukawa et al. 2009). Figure 2 shows the AKARI $9 \mu \mathrm{m}$ and $18 \mu \mathrm{m}$ composite map of RCW49, where the central region is saturated in the $18 \mu \mathrm{m}$ band. In the figure, two examples of the AKARI near-IR 2-5 $\mu \mathrm{m}$ slit spectra are shown together with their observational positions, from each of which a $3.3 \mu \mathrm{m}$ emission is detected. However we should be careful about this interpretation because the $3.3 \mu \mathrm{m}$ emission is also caused by the hydrogen recombination $\operatorname{Pf} \delta$ line at $3.30 \mu \mathrm{m}$ overlapping with the PAH $3.3 \mu \mathrm{m}$ band. Because the intensity of the Pf $\delta$ line should be $\sim 0.7 \times \operatorname{Pf} \gamma$ for the case B condition, the $3.3 \mu \mathrm{m}$ emission is most likely dominated by the PAH emission only if the $3.3 \mu \mathrm{m}$ line is much stronger than the $\operatorname{Pf} \beta$ and $\operatorname{Pf} \gamma$ lines at $4.66 \mu \mathrm{m}$ and $3.75 \mu \mathrm{m}$, respectively. By taking this into account, the presence of the PAH $3.3 \mu \mathrm{m}$ emission with its subfeatures at $3.4-3.5 \mu \mathrm{m}$ is confirmed in many regions for which spectroscopic observations have been performed around Wd2 except for its very central region. This physically reflects the presence of very small PAHs because PAHs with the number of C-atoms $>100$ or the size $>6 \AA$ cannot emit the $3.3 \mu \mathrm{m}$ feature efficiently (Draine \& Li 2007). It is thus found that even very small PAHs survive for $\sim 4 \times 10^{6}$ years under intense UV radiation field near very active star-forming region, which shows a striking contrast to the SNRs where PAHs are very easily destroyed.

In general, suppressed PAH emission relative to dust emission (e.g. unusually low ratios of the AKARI $9 \mu \mathrm{m}$ to $18 \mu \mathrm{m}$ band intensity) may serve as evidence of shock gas heating rather than radiative heating. For example, the NANTEN CO observations by Fukui et al. (2006) revealed the presence of large-scale molecular loops in the Galactic center region. The theoretical simulation by Machida et al. (2009) predicted magnetic loops can buoyantly rise due to the Parker instability 


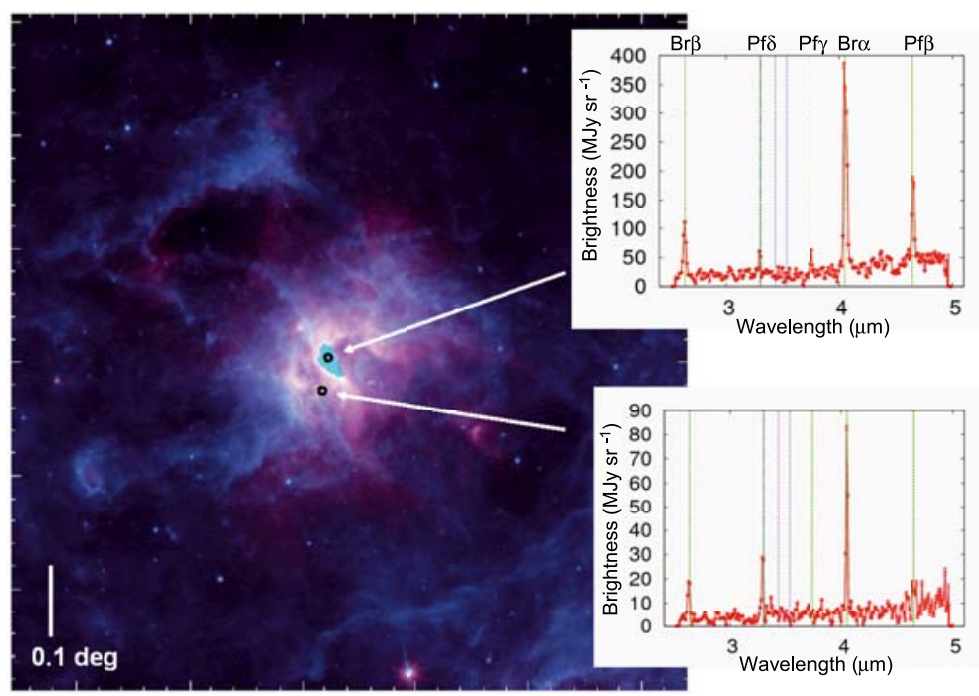

Fig. 2. AKARI $9 \mu \mathrm{m}$ (blue) and $18 \mu \mathrm{m}$ (red) composite image of the RCW49 region, where the center is saturated in the $18 \mu \mathrm{m}$ band. Two examples of AKARI near-IR 2$5 \mu \mathrm{m}$ slit spectra are shown together with their observational positions.

with disk gas, which cools at the top and then slides down along the loop with a speed of $\sim 30 \mathrm{kms}^{-1}$, presumably resulting in shock gas heating. If this is the case, we can expect that PAH emission is relatively suppressed at the foot points of the molecular loops.

\section{PAHs in the haloes of external galaxies}

Several authors have reported the detection of PAH emission in the haloes of nearby edge-on galaxies. As for relatively quiescent normal galaxies, PAH emissions in the haloes of NGC 5529 and NGC 5907 extending up to $\sim 10 \mathrm{kpc}$ and $\sim 6 \mathrm{kpc}$ above the disks were detected with ISO by Irwin et al. (2007) and Irwin \& Madden (2006), respectively. The latter was the first report of the detection of PAHs in the halo of an external galaxy. For NGC 5907, Irwin \& Madden (2006) found that the ratio of the PAH 11.3 to 7.7 band increases with distance from the galactic plane and also pointed out that there is a similarity in the spatial distribution between the PAH $6.3 \mu \mathrm{m}$ and $\mathrm{H} \alpha$ emission. Whaley et al. (2009) showed the presence of PAH emission in the halo of NGC 891 extending up to $\sim 3 \mathrm{kpc}$ above the disk with Spitzer and ISO. They compared the vertical extents of various components and found that the cool dust extends to larger scale heights than the PAHs and warm dust.

For more active galaxies, PAH emission from the galactic superwind of M 82 was observed with Spitzer and was found to widely extend around the halo up to 

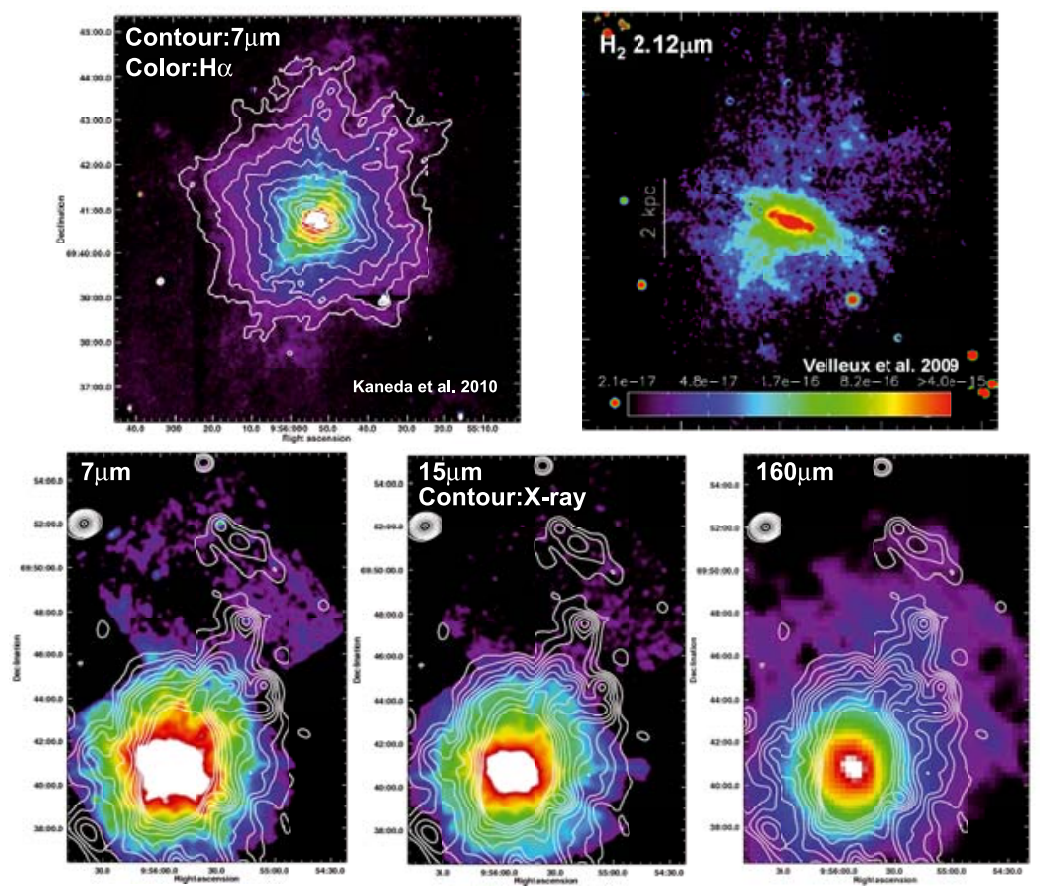

Fig. 3. AKARI $7 \mu \mathrm{m}$ band contour map of M 82 overlaid on the continuum-subtracted $\mathrm{H} \alpha$ image (Kaneda et al. 2010a) on the left, and $\mathrm{H}_{2} 2.12 \mu \mathrm{m}$ emission image (Veilleux et al. 2009) on the right of the upper panel. In the lower panel, the low-level $7 \mu \mathrm{m}$, $15 \mu \mathrm{m}$, and $160 \mu \mathrm{m}$ band images of M 82 including the Cap region, overlaid on the XMM/Newton X-ray (0.2-10 keV) contour map (Kaneda et al. 2010a).

$\sim 6 \mathrm{kpc}$ from the center (Engelbracht et al. 2006; Beirão et al. 2008). However one serious problem is that the central starburst core is dazzlingly bright due to tremendous star-forming activity in the central region of M 82 (Telesco \& Harper 1980); indeed M 82 is the brightest galaxy in the mid- and far-IR after the Magellanic Clouds. Then, instrumental effects caused by saturation made quantitative discussion about the low-surface brightness distribution around the halo rather difficult. Tacconi-Garman et al. (2005) performed high-spatial observations of the central region of NGC 253 with the IR camera and spectrograph ISAAC on ESO Very Large Telescope UT1. They found the presence of PAH emission in the galactic superwind of NGC 253 extended on a $0.1 \mathrm{kpc}$ scale on the basis of the $3.28 \mu \mathrm{m}$ narrow $(0.05 \mu \mathrm{m}$ width) band image, for which, however, the contribution by the $\operatorname{Pf} \delta$ line may have to be taken into account as mentioned above.

AKARI also observed M 82 over a wide area from the center to the halo in the mid- and far-IR in deep pointed observations (Kaneda et al. 2010a). One of the AKARI merits is a large dynamic range of signal detection; AKARI has 
a special mode to observe bright sources so that the instruments may not suffer saturation effects. The AKARI M 82 data consist of four mid-IR narrow-band images at reference wavelengths of $7 \mu \mathrm{m}$ (effective band width: $1.75 \mu \mathrm{m}$ ), $11 \mu \mathrm{m}$ $(4.12 \mu \mathrm{m}), 15 \mu \mathrm{m}(5.98 \mu \mathrm{m})$, and $24 \mu \mathrm{m}(5.34 \mu \mathrm{m})$, the allocation of which is ideal to discriminate between the PAH emission features $(7 \mu \mathrm{m}$ band tracing the PAH 7.7 and $8.6 \mu \mathrm{m}$ features, $11 \mu \mathrm{m}$ band tracing the 11.3 and $12.7 \mu \mathrm{m}$ features) and the MIR dust continuum emission $(15 \mu \mathrm{m}, 24 \mu \mathrm{m})$. The data also include four far-IR photometric bands; 2 wide bands at central wavelengths of $90 \mu \mathrm{m}$ (effective band width: $37.9 \mu \mathrm{m})$ and $140 \mu \mathrm{m}(52.4 \mu \mathrm{m})$ and 2 narrow bands at $65 \mu \mathrm{m}$ $(21.7 \mu \mathrm{m})$ and $160 \mu \mathrm{m}(34.1 \mu \mathrm{m})$. The left upper panel of Figure 3 shows the AKARI $7 \mu \mathrm{m}$ band contour map of M 82 for a $10 \times 10 \operatorname{arcmin}^{2}$ area, which reveals filamentary structures widely and faintly extended into the halo. As compared to the $\mathrm{H} \alpha$ distribution in color, a very tight correlation is found between the PAHs and the ionized superwind, providing evidence that the PAHs are well mixed in the ionized superwind gas and outflowing from the disk. In contrast, the $\mathrm{H}_{2} 2.12 \mu \mathrm{m}$ emission (Veilleux et al. 2009) shows a relatively loose correlation with the PAH emission especially in the northern halo (the right upper panel of Fig. 3). The deprojected outflow velocity of the $\mathrm{H} \alpha$ filaments is $525-655 \mathrm{~km} \mathrm{~s}^{-1}$ (Shopbell \& Bland-Hawthorn 1998). Therefore the PAHs seem to have survived in such a harsh environment for about $5 \times 10^{6}$ years to reach the observed positions, $\sim 3 \mathrm{kpc}$ above the disk, in the ionized superwind but not X-ray-emitting hot plasma because the destruction time in a hot plasma should be very short, $\sim 100$ years (Micelotta et al. 2010b).

Far beyond the disk of M 82, there is a region called X-ray Cap. The lower panel of Figure 3 shows the AKARI wide-area images of M 82 covering the Cap region at $7 \mu \mathrm{m}, 15 \mu \mathrm{m}$, and $160 \mu \mathrm{m}$, exhibiting the low-brightness distributions of the PAH, warm dust, and cool dust emission, respectively. The X-ray contours are overlaid for each image. Hence PAH emission is widely extended in the Cap region as well, which is presumably caused by the past tidal interaction of M 82 with M 81 about $10^{8}$ years ago (Walter et al. 2002). There is some anticorrelation between the X-ray and PAH emission at the Cap position and also at the upstream of the X-ray superwind. The $15 \mu \mathrm{m}$ warm dust emission is reasonably weak far beyond the disk, and the $160 \mu \mathrm{m}$ band clearly shows emission extended to the same direction as the X-ray superwind. Therefore the cool dust is also outflowing, entrained by the hot plasma. By comparing these maps, it is found that the destruction of PAHs in the X-ray plasma is clearly faster than the destruction of far-IR dust.

Another clear example of PAH detection from galactic superwinds is obtained for NGC 1569, a starburst dwarf galaxy possessing a very young (starburst peaked $\sim 5 \times 10^{6}$ years ago) and low-metalicity $\left(Z \sim 1 / 4 Z_{\odot}\right)$ environment; thus we expect a very small contribution from old stars to interstellar PAHs (Galliano et al. 2008). The background-subtracted spectra obtained by AKARI revealed the presence of the $3.3,6.2,7.7$, and $11.3 \mu \mathrm{m}$ PAH emission features in the prominent X-ray $/ \mathrm{H} \alpha$ filament produced by the galactic superwinds of NGC 1569 (Onaka et al. 2010). The filament age is approximately $10^{6}$ years, whereas the destruction timescale 

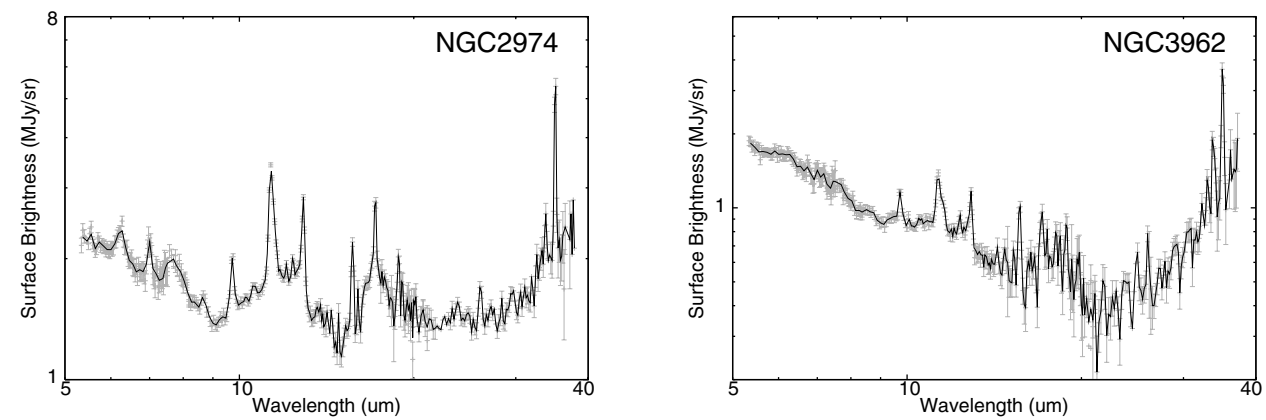

Fig. 4. Mid-IR spectra of the centers of the elliptical galaxies NGC 2974 and NGC 3962 obtained by the Spitzer/IRS staring observations. The figures are taken from Kaneda et al. (2008).

of small PAHs must be shorter than 100 years. To reconcile the discrepancy, we expect that PAHs may be produced by fragmentation of carbonaceous grains in shocks (Onaka et al. 2010).

\section{PAHs in elliptical galaxies}

Elliptical galaxies provide a unique environment: little UV from old stars and interstellar space filled by hot plasma, which are hostile conditions for PAH emission. Nevertheless, the early study with ISO by Xilouris et al. (2004) showed a hint of PAH emission in the spectral energy distributions of 10 elliptical galaxies out of 18. Then Spitzer/IRS clearly detected PAH emissions from the spectra of 14 elliptical galaxies out of 18 galaxies (Kaneda et al. 2008). Figure 4 shows such examples, where the prominent $11.3 \mu \mathrm{m}$ and the broad $17 \mu \mathrm{m}$ feature can be seen while usually the strongest $7.7 \mu \mathrm{m}$ feature is rather weak. The relative weakness of the $7.7 \mu \mathrm{m}$ feature is almost commonly observed among the elliptical galaxies. Since the $11.3 \mu \mathrm{m}$ feature is attributed to a $\mathrm{C}-\mathrm{H}$ out-of-plane bending mode mainly arising from neutral PAHs (e.g. Allamandola et al. 1989), a natural interpretation is that neutral PAHs are dominant over ionized PAHs due to soft radiation field from old stars, which is typical of elliptical galaxies. As another case supporting this interpretation, from a systematic study of mid-IR spectra of Galactic regions, Magellanic HII regions, and galaxies of various types (dwarf, spiral, starburst) by ISO and Spitzer, Galliano et al. (2008) found that the 6.2, 7.7, and $8.6 \mu \mathrm{m}$ bands are essentially tied together, while the ratios between these bands and the $11.3 \mu \mathrm{m}$ band vary by one order of magnitude. They concluded that the relative variations of the band ratios are mainly controlled by the fraction of ionized PAHs.

Alternatively, the characteristics of the PAH emission may be regulated by nuclear activities of the galaxies. A significant fraction of local elliptical galaxies are known to harbor a low-luminosity AGN (LLAGN), including a low-ionization nuclear emission region (LINER) nucleus (e.g. Ho et al. 1997). Smith et al. (2007) 


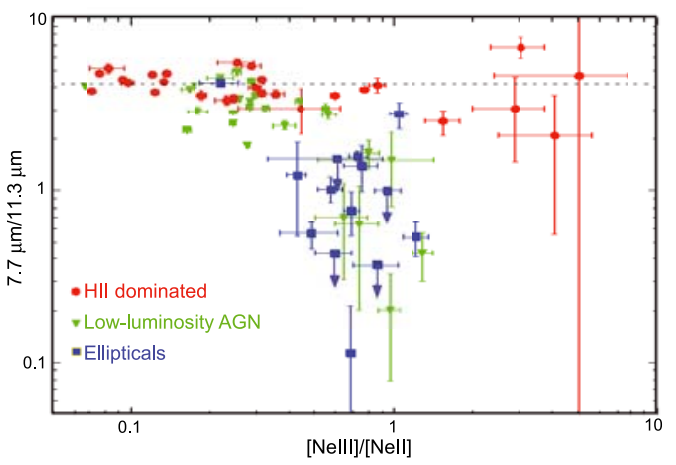

Fig. 5. PAH $7.7 \mu \mathrm{m} / 11.3 \mu \mathrm{m}$ ratios plotted against the line ratios of [NeIII]/[NeII] taken from Smith et al. (2007) for the galaxies with HII-dominated nuclei and LLAGNs, and from Kaneda et al. (2008) for the elliptical galaxies.

have reported that the peculiar PAH features with an unusually low ratio of the $7.7 \mu \mathrm{m}$ to the $11.3 \mu \mathrm{m}$ emission strength arise from systems with relatively hard radiation fields of LLAGNs. Figure 5 shows a plot of PAH $7.7 \mu \mathrm{m} / 11.3 \mu \mathrm{m}$ versus $[\mathrm{NeIII}] /[\mathrm{NeII}]$ taken from Smith et al. (2007) and the elliptical galaxy data from Kaneda et al. (2008) are plotted together. Generally, the $[\mathrm{NeIII}] /[\mathrm{NeII}]$ is an indicator of radiation hardness, and the elliptical galaxy data seem to follow a global trend. If this trend is true, PAHs in the elliptical galaxies may be affected by hard radiation from the LINER nuclei through selective destruction of smaller PAHs. Indeed, similar weakness of PAH features in the $5-10 \mu \mathrm{m}$ range relative to strong $11.3 \mu \mathrm{m}$ features were also obtained for radio galaxies (Leipski et al. 2009) and IR-faint LINERs (Sturm et al. 2006).

Recent deep spectral mapping with Spitzer/IRS clearly reveals the spatial distributions of PAH emission in elliptical galaxies for the first time (Kaneda et al. 2010b). An exemplary case, NGC 4589, is shown in Figure 6, where the upper panel displays the background-subtracted spectra of the central $15^{\prime \prime}$ region with the slit positions overlaid on the optical image. The lower panel of Figure 6 shows the distribution of the PAH $11.3 \mu \mathrm{m}$ emission in color as well as that of the $5.5-6.5 \mu \mathrm{m}$ continuum emission in contours. The $5.5-6.5 \mu \mathrm{m}$ continuum emission shows a smooth stellar distribution, which is consistent with the 2MASS image in the upper panel of Figure 6. However, the PAH $11.3 \mu \mathrm{m}$ emission exhibits a distinctly elongated distribution, posessing an excellent spatial coincidence with the minor-axis optical dust lane (see Fig. 2 of Möllenhoff \& Bender 1989). Kaneda et al. (2010b) also showed that the molecular hydrogen line emissions come from the dust lane, and that the [NeII] line emission shows a more compact distribution near the nucleus than the PAH features. Thus the PAH emission comes predominantly from dense gas in the optical dust lane, where the properties of the PAHs are not significantly regulated by hard radiation from the LINER nucleus. Since 

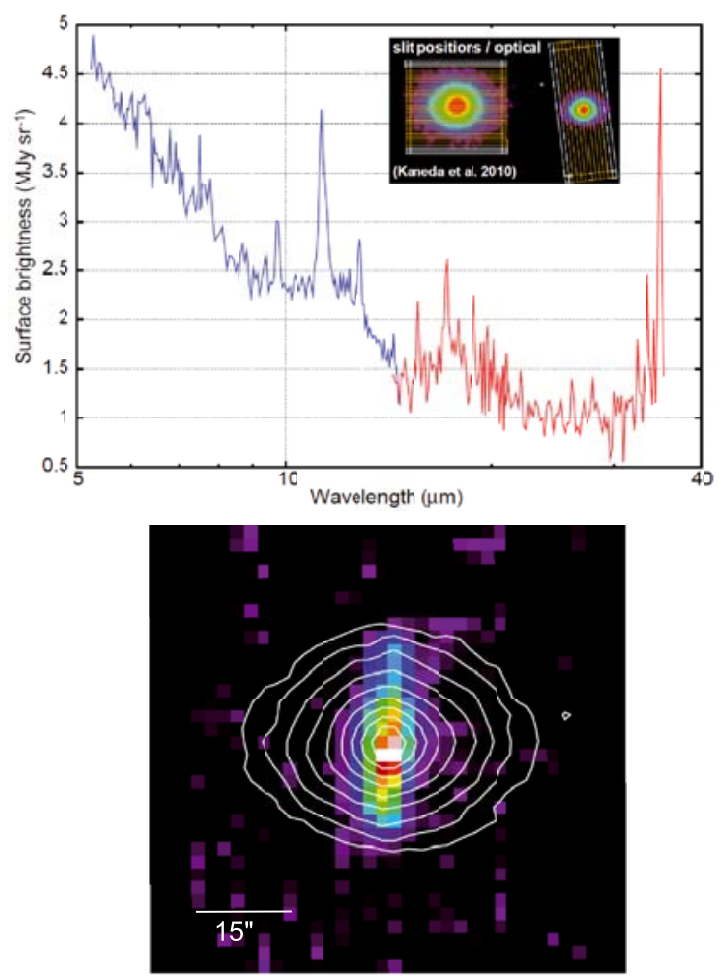

Fig. 6. Upper panel: the background-subtracted IRS SL (blue) and LL (red) spectra of the central $15^{\prime \prime}$ region of NGC 4589. The inset shows the SL (left) and LL (right) slit positions for the spectral mapping, superposed on the 2MASS image. Lower panel: the distribution of the PAH $11.3 \mu \mathrm{m}$ emission in color as well as that of the $5.5-6.5 \mu \mathrm{m}$ continuum emission in contours. For details, see Kaneda et al. (2010b).

NGC 4589 is thought to be an about $10^{8}$ year old merger (Kawabata et al. 2010), the result may reflect that the PAHs are secondary products from the evolution of the dust brought in by a merger.

As for the signature of interaction of PAHs with hot plasma, no correlation has been found between PAH and X-ray emission among elliptical galaxies, which implies that a large fraction of observed PAHs are not continuously supplied into the interstellar space from old stars, but sporadically from other internal or external sources. A possible major origin of the PAHs is merger events as suggested by the above case. Another interesting possibility was suggested by Temi et al. (2007) such that the AGN-assisted feedback outflow from a central dust reservoir may supply PAHs into the interstellar space of elliptical galaxies through residual dust fragments diminished by sputtering. 


\section{Summary and future prospects}

We extensively review observational results of PAH emission in harsh interstellar environments. The observations have indicated that PAHs in the shock regions of SNRs are destroyed very easily on 10-100 year timescales (e.g. Tycho SNR), where dust grains survive for a much longer time. There are probably a few exceptional cases (3C 396 and N132D in LMC) where SNR still show PAH emission. In contrast, PAHs survive for $10^{6}-10^{7}$ timescales under very intense UV radiation field in RCW49, one of the most massive star clusters in our Galaxy. The observations have also shown that PAHs are present in the ionized galactic superwinds of starburst galaxies. For M 82, PAHs are widely spread around the halo through the galactic superwind and also probably through past tidal interaction with M 81 . For NGC 1569, even very small PAHs emitting the $3.3 \mu \mathrm{m}$ feature exist in the prominent $\mathrm{H} \alpha$ superwind. Finally the observations have revealed that PAHs are present in many elliptical galaxies. The PAH emission from elliptical galaxies is characterized by unusually low ratios of the $7.7 \mu \mathrm{m}$ to the $11.3 \mu \mathrm{m}$ and $17 \mu \mathrm{m}$ emission features, probably reflecting that neutral PAHs are dominant over ionized PAHs due to very soft radiation field from old stars. In particular, for NGC 4589, the PAH emission is found to predominantly come from the optical dust lane. No signatures of interaction between PAHs and hot plasmas have been found among elliptical galaxies, which implies that a large fraction of observed PAHs are sporadically supplied into the interstellar space from internal or external sources other than from old stars.

In future, SPICA (Space Infrared Telescope for Cosmology and Astrophysics; Swinyard et al. 2009) will be launched around 2018, covering the wavelength range from 5 to $200 \mu \mathrm{m}$ by imaging spectroscopy with a 3-m aperture, $6 \mathrm{~K}$ telescope. Detailed studies of PAH emission in the far-IR regime, such as the ro-vibrational modes (see Joblin et al. in this volume), can be carried out with SPICA, which are critical to trace the structural changes of PAH molecules being processed in harsh environments.

Most of the researches presented in this paper are based on observations made with the Spitzer Space Telescope, which is operated by the Jet Propulsion Laboratory, Carlifornia Institute of Technology under NASA contract 1407, and AKARI, a JAXA project with the participation of ESA.

\section{References}

Allain, T., Leach, S., \& Sedlmayr, E., 1996, A\&A, 305, 602

Allamandola, L.J., Tielens, A.G.G.M., \& Barker, J.R., 1989, ApJS, 71, 733

Bendo, G.J., Draine, B.T., Engelbracht, C.W., et al., 2008, MNRAS, 389, 629

Beirão, P., Brandl, B.R., Appleton, P.N., et al., 2008, ApJ, 676, 304

Boulanger, F., Beichman, C., Désert, F.X., et al., 1988, ApJ, 332, 328

Désert, F.X., Boulanger, F., \& Puget, J.L., 1990, A\&A, 237, 215

Draine, B.T., \& Li, A., 2007, ApJ, 657, 810 
Engelbracht, C.W., Kundurthy, P., Gordon, K.D., et al., 2006, ApJ, 642, L12

Fukui, Y., Yamamoto, H., Fujishita, M., et al., 2006, Science, 314, 106

Furukawa, N., Dawson, J.R., Ohama, A., et al., 2009, ApJ, 696, 115

Galliano, F., Madden, S.C., Tielens, A.G.G.M., Peeters, E., \& Jones, A.P., 2008, ApJ, 679,310

Ho, L.C., Filippenko, A.V., \& Sargent, W.L.W., 1997, ApJ, 487, 579

Irwin, J.A., Kennedy, H., Parkin, T., \& Madden, S.C., 2007, A\&A, 474, 461

Irwin, J.A., \& Madden, S.C., 2006, A\&A, 445, 123

Ishihara, D., Kaneda, H., Furuzawa, A., et al., 2010, A\&A, 521, L61

Jones, A.P., Tielens, A.G.G.M., \& Hollenbach, D.J., 1996, ApJ, 469, 740

Kaneda, H., Onaka, T., Sakon, I., et al., 2008, ApJ, 684, 270

Kaneda, H., Ishihara, D., Suzuki, T., et al., 2010a, A\&A, 514, A14

Kaneda, H., Onaka, T., Sakon, I., et al., 2010b, ApJ, 716, L161

Kawabata, K.S., Maeda, K., Nomoto, K., et al., 2010, Nature, 465, 326

Leipski, C., Antonucci, R., Ogle, P., \& Whysong, D., 2009, ApJ, 701, 891

Machida, M., Matsumoto, R., Nozawa, S., et al., 2009, PASJ, 61, 411

Micellota, E.R., Jones, A.P., \& Tielens, A.G.G.M., 2010a, A\&A, 510, A36

Micellota, E.R., Jones, A.P., \& Tielens, A.G.G.M., 2010b, A\&A, 510, A37

Möllenhoff, C., \& Bender, R., 1989, A\&A, 214, 61

Neufeld, D.A., Hollenbach, D.J., Kaufman, M.J., et al., 2007, ApJ, 664, 890

Onaka, T., Matsuhara, H., Wada, T., et al., 2007, PASJ, 59, S401

Onaka, T., Matsumoto, H., Sakon, I., \& Kaneda, H., 2010, A\&A, 514, A15

Peeters, E., Mattioda, A.L., Hudgins, D.M., \& Allamandola, L.J., 2004, ApJ, 617, L65

Reach, W.T., Rho, J., Tappe, A., et al., 2006, AJ, 131, 1479

Reynoso, E.M., Moffett, D.A., Goss, W.M., et al., 1997, ApJ, 491, 816

Shopbell, P.L., \& Bland-Hawthorn, J., 1998, ApJ, 493, 129

Smith, J.D.T., Draine, B.T., Dale, D.A., et al., 2007, ApJ, 656, 770

Sturm, E., Rupke, D., Contursi, A., et al., 2006, ApJ, 653, L13

Swinyard, B. Nakagawa, T., Merken, P., et al., 2009, Experimental Astronomy, 23, 193

Tacconi-Garman, L.E., Sturm, E., Lehnert, M., et al., 2005, A\&A, 432, 91

Tappe, A., Rho, J., \& Reach, W.T., 2006, ApJ, 653, 267

Telesco, C.M., \& Harper, D.A., 1980, ApJ, 235, 392

Temi, P., Brighenti, F., \& Mathews, W.G., 2007, ApJ, 666, 222

Tielens, A.G.G.M., 2008, ARA\&A, 46, 289

Van Kerckhoven, C., Hony, S., Peeters, E., et al., 2000, A\&A, 357, 1013

Veilleux, S., Rupke, D.S.N., \& Swaters, R., 2009, ApJ, 700, L149

Walter, F., Weiss, A., \& Scoville, N., 2002, ApJ, 580, L21

Warren, J.S., Hughes, J.P., Badenes, C., et al., 2005, ApJ, 634, 376

Whaley, C.H., Irwin, J.A., Madden, S.C., Galliano, F., \& Bendo, G.J., 2009, MNRAS, 395, 97

Xilouris, E.M., Madden, S.C., Galliano, F., Vigroux, L., \& Sauvage, M., 2004, A\&A, 416,41 